\section{International Scientific Journal Theoretical \& Applied Science}

p-ISSN: 2308-4944 (print) e-ISSN: 2409-0085 (online)

Year: 2018 Issue: 09 Volume: 65

Published: $29.09 .2018 \quad$ http://T-Science.org

SECTION 20. Medicine
Vusala Zahid Khalilova

the doctor-laboratory assistant of the Oncological Clinic, of the Azerbaijan Medical University

Arif Mustafa Efedyev

Doctor of Medical Sciences, Head of the Department of Biochemistry

Azerbaijan Medical University

Gulnara Alicha Jafarova Candidate of Biological Sciences, Senior Researcher of the research laboratory of the biochemical department, doctor-laboratory assistant of the Oncological clinic of the Azerbaijan Medical University

Nasimi Veli Gasimov Candidate of Medical Sciences, Chief Doctor of Oncology Clinic Azerbaijan Medical University

\title{
CHANGE IN SOME BIOCHEMICAL INDICATORS IN WOMEN WITH ONCOLOGICAL DISEASES OF THE REPRODUCTIVE SYSTEM
}

\begin{abstract}
This study included 47 patients. All the examined patients were divided into three groups: 1) group benign neoplasms (23); 2) group - malignant tumors (15); 3) group - metastatic tumors. 15 healthy people were included in the control group. The concentration of CA125 in the group of women with cancer of the female reproductive system was significantly increased $(205.1 \pm 16.83 \mathrm{ng} / \mathrm{ml})$ in comparison with the group of practically healthy people $(12.8 \pm 0.06 \mathrm{ng} / \mathrm{ml})$. The data obtained suggest that the increase in CA125 in the serum of patients with malignant tumors is important in assessing the aggressiveness of the process. In patients with malignant tumors, there was a significant increase in $I L-8$ and TNF- $\alpha$, as well as the activity of $A L P, A L T, A C T, G G T$ in the serum compared to benign tumors with metastases. The data presented by us indicate that CA125, IL-8, TNF- $\alpha$ and ALP activity are closely related to the pathogenic mechanisms of malignant tumor growth.
\end{abstract}

Key words: cancer of the reproductive system of women, CEA, CA125, inteleikin-8, tumor necrosis factor, alkaline phosphatase.

Language: Russian

Citation: Khalilova VZ, Efedyev AM, Jafarova GA, Gasimov NV (2018) CHANGE IN SOME BIOCHEMICAL INDICATORS IN WOMEN WITH ONCOLOGICAL DISEASES OF THE REPRODUCTIVE SYSTEM. ISJ Theoretical \& Applied Science, 09 (65): 264-268.

Soi: http://s-o-i.org/1.1/TAS-09-65-44 Doi: crostef https://dx.doi.org/10.15863/TAS.2018.09.65.44

\section{ИЗМЕНЕНИЕ НЕКОТОРЫХ БИОХИМИЧЕСКИХ ПОКАЗАТЕЛЕЙ У ЖЕНЩИН ОНКОЛОГИЧЕСКИМИ ЗАБОЛЕВАНИЯМИ РЕПРОДУКТИВНОЙ СИСТЕМЫ}

Аннотация: В настоящеее исследование включено 47 пациентов. Все обследованные больные были объединень в три группь:: 1) группа - доброкачественные новообразования (23); 2) группа злокачественные опухоли (15); 3) группа - метастатические опухоли. В группу контроля было включено 15 практически здоровых людей. Концентрация СА125 в группе больных раком женской репродуктивной системы $(205,1 \pm 16,83$ нг/мл) была достоверно повышена по сравнению с группой практически здоровых

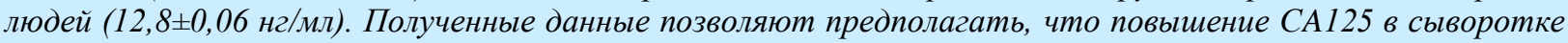
крови больных злокачественными опухолями имеет важное значение при оченке агрессивности прочесса. У больных со злокачественными опухолями наблюдалось значительное повышение ИЛ-8 и ФНО- $\alpha$, а также активности ЩФ, АЛТ, АСТ, ГГТ в сыворотке крови по сравнению доброкачественными новообразованиями с метастазами. Представленные нами данные указывают, что СА125, ИЛ-8, ФНО- $а$ активность ЩФ тесно связаны с патогенетическими механизмами роста злокачественньх опухолей.

Ключевые слова: рак репродуктивной системы женщин, СЕА, СА125, интелейкин-8, фактор некроза опухоли, щелочная фосфатаза. 


\section{Введение}

Сегодня раковые болезни репродуктивной системы составляет приблизительно 15\% от всех злокачественных новообразований у женщин. По частоте на первом месте стоит рак эндометрия, затем - яичника и третье место занимает рак шейки матки [1]. Однако по смертности рак яичника занимает ведущее место. Отсутствие ранних симптомов и своевременного диагноза приводит к тому, что примерно 70\% больных

Поздняя диагностика рака репродуктивной системы и бессимптомное течение усугубляет клинику болезни. Появление боли в последней стадии, кровотечение, рост лимфатических узлов, заметный рост и распространение размера опухоли, наличие близких и поздних метастазов и подобные симптомы усложняют проблему [3]. Установлено, что доброкачественные заболевания органов гениталий играют важную роль в развитии злокачественных новообразований. Сегодня наиболее распространенным среди гинекологических заболеваниях являются миома матки. Миома является доброкачественной гормонозависимой опухолью эндометрии. Клинические признаки наблюдается только тогда, когда опухоль растёт и вытесняет окружающие ткани. У более молодых женщин обычно высокая вероятность появления миомы в возрасте 30-45 лет. Во время менопаузы наблюдается уменьшение размера или даже иногда полное исчезновение опухоли. Это доброкачественное новообразование появляется на стенке матки и прогрессирование опухоли приводить к увеличению его размеров. Хотя этиология фибромы до сих пор не полностью изучена, но многие исследователи считают, что в возникновении опухоли играют важную роль гормональная чувствительность к эстрогену и генетические факторы. Поскольку гормональная зависимость является преобладающей в образовании опухоли, она не встречается у девочек до менструального цикла и у женщин в постклимактерический период. Дисплазия клеток и тканей приводить прогрессированию предракового состояния и образованию злокачественных опухолей, поэтому к этим патологиям нужно относиться серьёзно $[4,5,6]$. Несмотря на развитию современных методов диагностики и лечения этих опухолей, он попрежнему остаётся одной из наиболее важной и актуальной проблемой в области онкологии.

Симптомы болезни на ранних стадиях являются неспецифическими, и поэтому для точной диагностики злокачественных опухолей необходимы проведение компьютерной и магнитно-резонансной томографии, различных клинических, радиологических, морфологических и лабораторных тестов. Но некоторые методы выявления патологии раком яичника и шейки матки при обращении имеют распространенную стадию заболевания. Поздняя диагностика заболевания и неадекватное лечение приводить к прогрессированию заболевания и развитию метастаза, также увеличивает смертность среди больных с онкологическими заболеваниями репродуктивной системы. Средняя 5-летняя выживаемость около $30 \%$, при 3 и 4-ой стадиях заболевания - эта цифра достигает лишь 10\% [2].

эндометрия, как биопсия и гистероскопия являются инвазивными и недостаточными для ранней диагностики [5].

Исследования CA125 и CEA у больных с опухолями репродуктивной системы женщин считаются одним из наиболее важных показателей определения злокачественности. Антиген СА125 представляет собой глюкопротеин и имеет молекулярную массу 220 кДа. Он обычно встречается в слизистой оболочке шейки матки-эндометрии. Он также содержится в поджелудочной железе, жёлчном пузыре, желудке, бронхах, почках и кишечнике. Доказано, что концентрация СА 125 повышена у 65-95\% больных раком яичников и шейки матки. Концентрация СА125 увеличивается не только при злокачественных заболеваниях, также наблюдалось повышение его уровня при воспалительных заболеваниях различных органов репродуктивной системы, I триместре беременности и эндометриозе. Поэтому определение СА125 является недостаточно чувствительным и специфичным маркером при ранних стадиях болезни [7]. В последнее время значительное внимание уделяется роли цитокинам в патогенезе заболеваний репродуктивной системы. Доказано зависимость между распространенностью опухолевого процесса и цитокиновым профилем у больных раком репродуктивных органов $[8,9]$.

Прогрессирование болезни сопровождается метастазами опухоли в различным органов, в том числе в печени. К основным биохимическим маркерам метастатического поражения печени относятся: щелочная фосфатаза - ЩФ, трансаминазы, лактатдегидрогеназа (ЛДГ) и др [10].

Целью исследования является изучение уровня CEA, CA125 и некоторых биохимических показателей у пациентов со злокачественными опухолями женских гениталий.

Материалы и методы. С этой целью были исследованы венозная кровь 47 пациентов, которым был поставлен диагноз доброкачественный и злокачественный опухоль женской репродуктивной системы. Больным был поставлен диагноз в онкологической клинике АТУ в 2017 году. Контрольная группа составили 15 практически здоровых людей. Основываясь на 
клинических, инструментальных

И морфологических результатах, у 23 пациентов был выявлен доброкачественный, у 15-ти злокачественный, а 9-ти метастатический опухоль репродуктивной системы.

Во время исследования венозную кровь брали в чистую и сухую пробирку для получения сыворотки. Его выдерживали при комнатной температуре в течение 30 минут для образования сгустка. Затем центрифугируют в течение 15 минут.

Исследование концентрации СЕА и СЕА125 проводили с помошью метода иммуноферментного анализа (IFA) в автоматическом иммуноферментном анализаторе

Достоверность различий между средними значениями изученных параметров до и после лечения определяли $\mathrm{c}$ помощью $\mathrm{t}$ критерия Стьюдента и U-критерий Манна-Уитни. Достоверными считались различия при $\mathrm{p}<0,05$.
COBAS (Германия). Биохимические показатели (активность аланинаминотрансферазы (АЛТ), аспартатаминотрансферазы

(АCT), гаммаглютамилтрансферазы (ГГТ), щелочной фосфатазы (ЩФ) и концентрация креатина) были проанализированы с помощью реактивного набора и диагностической тест-системой: COBAS (Германия) на автоматическои анализатора (ROCHE).

Концентрацию интерлейкина-8 (ИЛ-8) и фактора некроза опухоли (ФНО- $\alpha)$ в сыворотке крови в исследуемых группах больных с помощью иммуноферментных тестсистем, «Вектор-Бест» (Россия) на иммуноферментном анализаторе «СтатФакс» .

Результаты и обсуждения. Результаты иммуноферментных исследований СЕА, СА125, ИЛ-8 и ФНО- $\alpha$ показаны в таблице 1.

Уровень СЕА vә СА125 у больных с заболеваниями репродуктивной системы

Таблица 1

\begin{tabular}{|c|c|c|c|c|}
\hline \multirow[b]{2}{*}{ Показатели } & \multicolumn{4}{|c|}{ Группы } \\
\hline & $\begin{array}{c}\text { Контроль } \\
(\mathrm{n}=15)\end{array}$ & $\begin{array}{c}\text { Доброкачественные } \\
\text { опухоли } \\
(\mathrm{n}=23)\end{array}$ & $\begin{array}{c}\text { Злокачественные } \\
\text { опухоли } \\
(\mathrm{n}=15)\end{array}$ & $\begin{array}{c}\text { Метастатические } \\
\text { опухоли } \\
(\mathrm{n}=9)\end{array}$ \\
\hline CEA ( & $\begin{array}{c}2,31 \pm 0,04 \\
(0,5-4,8)\end{array}$ & $\begin{array}{l}2,5 \pm 0,03 \\
(1,2-5,6)\end{array}$ & $\begin{array}{c}4,8 \pm 0,26 * * \\
(1,35-8,9)\end{array}$ & $\begin{array}{c}7,9 \pm 2,3 * * \\
(23,5-19,87)\end{array}$ \\
\hline CA125 (нг/ml) & $\begin{array}{l}12,8 \pm 0,06 \\
(5,2-29,7)\end{array}$ & $\begin{array}{c}13,27 \pm 0,11 \\
(8,24-26,84)\end{array}$ & $\begin{array}{c}205,1 \pm 16,83 * * * \\
(48,49-556,6)\end{array}$ & $\begin{array}{c}714,66 \pm 85,92 * * * \\
(287,2-3041)\end{array}$ \\
\hline $\begin{array}{l}\text { ИЛ-8, } \\
\text { Пг/мЛ }\end{array}$ & $\begin{array}{c}11,5 \pm 1,1 \\
(3,9-15,7)\end{array}$ & $\begin{array}{c}16,7 \pm 0,9 \\
(4,8-27,8)\end{array}$ & $\begin{array}{l}22,8 \pm 1,3 * \\
(6,9-31,9)\end{array}$ & $\begin{array}{c}32,7 \pm 1,7 * * \\
(8,1-39,8)\end{array}$ \\
\hline ФНО- $\alpha$, пг/мЛ & $\begin{array}{c}3,82 \pm 0,74 \\
(1,28-7,95)\end{array}$ & $\begin{array}{l}6,72 \pm 0,89 * * \\
(3,58-10,58)\end{array}$ & $\begin{array}{c}10,96 \pm 0,92^{* *} \\
(4,89-19,78)\end{array}$ & $\begin{array}{c}29,62 \pm 1,02 * * * \\
(7,54-39,83)\end{array}$ \\
\hline
\end{tabular}

Примечение: * - $<<0,05 ; * *$ - $\mathrm{p}<0,01 ; * * *-\mathrm{p}<0,001$ - по сравнению с контролем.

В результате исследования выявлено, что уровень СЕА и СА125 в крови у больных с доброкачественными опухолями репродуктивной системы почти не отличается с результатами практически здоровых людей. При злокачественных и метастатических опухолях содержание CEA и СА125 статистически достоверно увеличивается, соответственно, в 3,4 раза и 55,8 раза по сравнению с данными контрольной группы. Полученные данные свидетельствуют о том, что уровень СЕА и СА125 можно оценить в первую очередь для определения агрессивности процесса в опухолях женских половых путей. Мы считаем, что определение СА125 в опухоли женской генитальной системы имеет большое значение для дифференциальной диагностики между доброкачественными и злокачественными процессами.
Как показали результаты исследований, наблюдается повышение уровня ИЛ- 8 и ФНО- $\alpha$ в исследуемых группах относительно контрольной группы. Как оказалось, у больных с доброкачественными опухолями содержание ИЛ8 и ФНО- $\alpha$ увеличилось в 1,5 раза и 1,8 раза соответственно, по сравнению с контролем. У больных с злокачественными опухолями уровень ИЛ-8 и ФНО- $\alpha$ повысился в 1,9 и 2,9 раза; а метастатическими опухолями, соответственно в 2,8 и 7,8 раза по отношению к группе контроля. При злокачественных опухолях уровень возрастание ИЛ-8 и ФНО-а в крови было выражено в большей степени, чем при доброкачественных опухолях (таблица 2).

Повышение содержания в крови ИЛ-8 и ФНО- $\alpha$ свидетельствуют об активации системной неспецифической резистентности организма к развитию опухолевого процесса $[8,9]$. 


\begin{tabular}{|c|c|c|c|c|c|c|}
\hline Impact Factor: & $\begin{array}{l}\text { ISRA (India) } \\
\text { ISI (Dubai, UAE } \\
\text { GIF (Australia) } \\
\text { JIF }\end{array}$ & $\begin{array}{l}=1.344 \\
=0.829 \\
=0.564 \\
=1.500\end{array}$ & $\begin{array}{l}\text { SIS (USA) } \\
\text { PИНЦ (Russia) } \\
\text { ESJI (KZ) } \\
\text { SJIF (Morocco) }\end{array}$ & $\begin{array}{l}=0.912 \\
=0.156 \\
=4.102 \\
=\mathbf{5 . 6 6 7}\end{array}$ & $\begin{array}{l}\text { ICV (Poland) } \\
\text { PIF (India) } \\
\text { IBI (India) }\end{array}$ & $\begin{array}{l}=6.630 \\
=1.940 \\
=4.260\end{array}$ \\
\hline
\end{tabular}

Таблица 2

Результаты определения уровня биохимических показателей у больных с раком репродуктивной системы

\begin{tabular}{|c|c|c|c|c|}
\hline \multirow{2}{*}{ Группы } & \multicolumn{4}{|c|}{$\begin{array}{c}\text { Контроль } \\
(\mathrm{n}=15)\end{array}$} \\
\cline { 2 - 5 } & Контроль & $\begin{array}{c}\text { Доброкачественные } \\
\text { опухоли } \\
(\mathrm{n}=23)\end{array}$ & $\begin{array}{c}\text { 3локачественные } \\
\text { опухоли } \\
(\mathrm{n}=15)\end{array}$ & $\begin{array}{c}\text { Метастатические } \\
\text { опухоли } \\
(\mathrm{n}=9)\end{array}$ \\
\hline ЩФ (U/l) & $75,8 \pm 0,19$ & $80,36 \pm 17,64$ & $113,81 \pm 28,92^{* *}$ & $231,7 \pm 48,54^{* * *}$ \\
& $(50-136)$ & $(75,2-116,8)$ & $(1,71-167,2)$ & $(144,84-437)$ \\
\hline АЛТ (U/l) & $15,82 \pm 0,29$ & $16,85 \pm 0,25$ & $18,64 \pm 0,27$ & $94,83 \pm 11,95^{* *}$ \\
& $(10-42)$ & $(10,44-44,83)$ & $(12,13-34,33)$ & $(29,52-217,69)$ \\
\hline АСТ(U/l) & $13,48 \pm 0,15$ & $20,64 \pm 0,18$ & $23,22 \pm 0,19$ & $55,51 \pm 2,95^{* *}$ \\
& $(10-57)$ & $(12,25-27,29)$ & $(11,03-39,7)$ & $(29,33-157,9)$ \\
\hline ГГТ (U/л) & $20,8 \pm 0,12$ & $31,22 \pm 0,14$ & $34,85 \pm 0,28^{*}$ & $121,49 \pm 10,83^{* * *}$ \\
& $(5-32)$ & $(20,9-46,07)$ & $(26,1-58,85)$ & $(47,17-272,33)$ \\
\hline Креатинин & $0,53 \pm 0,07$ & $0,56 \pm 0,03$ & $0,63 \pm 0,09$ & $0,85 \pm 0,11^{*}$ \\
(мг/дл) & $(0,3-0,95)$ & $(0,43-0.84)$ & $(0,49-1,13)$ & $(0,58-1,42)$ \\
& & & & \\
\hline
\end{tabular}

Примечение: * - p<0,05; ** - p<0,01; *** - p<0,001 - по сравнению с контролем.

У больных доброкачественными опухолями не выявлено значительных изменений в биохимических значениях. Активность ЩФ, у больных доброкачественными опухолями незначительно отличалось от контрольной группы, но выявлено статистически значимое увеличение его активности при злокачественных опухолей. $\mathrm{y}$ больных злокачественными заболеваниями репродуктивной системы с метастазами установлено значительное повышение активности ЩФ, АЛТ, АСТ и ГГТ в $2 ; 5,1 ; 2,4$ и 5,8 раза, соответственно по сравнению с показателями больных без метастазов.

\section{Выводы}

Результаты исследования показывают, что уровни АЛТ, АСТ, ГГТ и ЩФ в сыворотке крови у пациентов с новообразованиями репродуктивной системы тесно связано с патогенезом и прогнозом заболевания. Определение этих показателей у больных обращавшиеся в клинику, может позволить дифференцировать процессы злокачественности и доброкачественности, а также обеспечить адекватное своевременное лечение.

Таким образом, высокая активность АЛТ, АСТ, ГГТ и ШФ в сыворотке пациентов с раком женских половых путей вызывает подозрение на метастазы. У пациентов со злокачественной опухолью определение СЕА, СА125, ИЛ-8, ФНО$\alpha$ и ЩФ позволяет идентифицировать биологические свойства опухоли.

\section{References:}

1. Aksel Yevqeniya Milyevna (2008) Statistika zlokacestvennix novoobrozovaniy jenskix polovix orqanov, Opuxoli jenskix polovix orqanov, N 1-2, p.76-80.

2. Ferlay J, Soerjomataram I, Ervik M, Dikshit R, Eser S, Mathers C et al. globocan 2012 v1.0, (2013), Cancer Incidence and Mortality Worldwide: IARC Cancer Base No. 11 Lyon, France: International Agency for Research on Cancer.
3. Schoell W.M., Janicek M.F., Mirhashemi R. (1999) Epidemiology and biology of cervical cancer // Semin. Surg. Oncol.-1999., Vol.16. №3. p. 203-211.

4. Kiselyev V.B. (2003) Virusi papilloma celoveka v razvitii raka cheyki matki, Moskva, p.90.

5. Morkhov K.Yu., Kuznetsov V.V. (2009) Rak cheyki matki v razvitii matki, Moskva, p.90., p. 199-225. 


\begin{tabular}{l|lr|ll|ll} 
& ISRA (India) & $=\mathbf{1 . 3 4 4}$ & SIS (USA) & $=\mathbf{0 . 9 1 2}$ & ICV (Poland) & $=\mathbf{6 . 6 3 0}$ \\
Impact Factor: & ISI (Dubai, UAE) $=\mathbf{0 . 8 2 9}$ & PUHЦ (Russia) $=\mathbf{0 . 1 5 6}$ & PIF (India) & $=\mathbf{1 . 9 4 0}$ \\
& GIF (Australia) & $\mathbf{0 . 5 6 4}$ & ESJI (KZ) & $=4.102$ & IBI (India) & $=\mathbf{4 . 2 6 0}$ \\
& JIF & $=\mathbf{1 . 5 0 0}$ & SJIF (Morocco) & $=2.031$ & & \\
\hline
\end{tabular}

6. Huiqin Guo and Kangla Tsung (2017) Tumor reductive therapies and antitumor immunity, Oncotarget.,Aug 15; 8(33): 55736-55749.

7. Hanikezi Tuerxun, Manrepa Tuerxun, Guzailinuer Maimaitituersun, Yilidana Yilihamu, Wuriguli Maimaitiming, Gulizapar Aierken, Zufeiya Aili (2018), Significance of serum CA-125 combined with CEA and HE-4 in diagnosis of epithelial ovarian cancer, Int $\mathbf{J}$ Clin Exp Med, No11(4), p.4156-4161.

8. PeterW. Szlosarek, MatthewJ. Grimshaw, Hagen Kulbe, Julia L. Wilson, George D. Wilbanks, Frances Burke and Frances, R. Balkwill (2006), Expression and regulation of tumor necrosis factor $\alpha$ in normal and malignant ovarian epithelium, Molecular cancer therapeutics, v.5 (2), p. 382-390.

9. Ellen C. Keeley, Borna Mehrad, Robert M. Strieter (2010) Chapter 3 - CXC Chemokines in Cancer Angiogenesis and Metastases, Advances in Cancer Research, v.106, p. 91-111.

10. Chenyue Zhang, Haiyong Wang, Zhouyu Ning, Litao Xu, Liping Zhuang, Peng Wang and Zhiqiang Meng (2017) Serum liver enzymes serve as prognostic factors in patients with intrahepatic cholangiocarcinoma, Onco Targets Ther., v 10, p.1441-1449. 